\title{
LEARNING ABOUT EDGE EFFECTS AND ULTRAMICROELECTRODES IN ELECTROCHEMISTRY: SYNERGY BETWEEN EXPERIMENTS AND SIMULATIONS
}

\author{
Magalí Gimeno $^{\mathrm{a}, \mathrm{b}}$ and Franco Martín Zanotto ${ }^{\mathrm{a}, \mathrm{b}, \mathrm{c}, *,(\mathbb{C})}$ \\ aDepartamento de Fisicoquímica, Facultad de Ciencias Químicas, Universidad Nacional de Córdoba, Ciudad Universitaria, \\ X5000HUA, Córdoba, Argentina \\ 'Instituto de Investigaciones en Fisicoquímica de Córdoba, CONICET, Ciudad Universitaria, X5000HUA, Córdoba, Argentina \\ 'Department of Chemistry and Biochemistry, Florida State University, 102 Varsity Drive, Tallahassee, FL 32306-4390, United \\ States of America
}

Recebido em 20/04/2020; aceito em 19/05/2020; publicado na web em 08/07/2020

\begin{abstract}
A short experimental activity aimed at upper level undergraduate students or beginner researchers is presented to introduce the concepts of edge effects and hemispherical diffusion at ultramicroelectrodes. Students will perform simple cyclic voltammetry experiments varying the scan rate and using disk electrodes with different radii. They are then asked to interpret the results based on their previous knowledge. Simulations are also performed, which provide a clear visual interpretation of the underlying diffusion process and show the transition between linear and hemispherical diffusion regimes. The simulations are also presented as a tool to extend the study beyond the range of experimentally accessible conditions. The interplay between voltammetry experiments and simulations allows a natural comprehension of these concepts without the need of a previous rigorous theoretical introduction.
\end{abstract}

Keywords: computer simulations; electrochemistry; voltammetry; diffusion.

\section{INTRODUCTION}

Cyclic voltammetry is usually the go-to technique when exploring a new electrochemical system due to the large amount of information it yields, its simplicity, versatility, ease of use and low cost. ${ }^{1-3}$ When introducing this technique, naturally the well-known simple oneelectron reversible voltammogram for a macroscopic electrode is usually discussed, which allows the student or reader to familiarize with the main phenomena involved in a voltammetry experiment. Subsequently, additional aspects are included, such as solution resistance, irreversible or quasi-reversible electron transfers, coupled chemical reactions, adsorption, two-electron processes, etc..$^{1,3-7}$

Recently, an excellent comprehensive beginners' guide for cyclic voltammetry was published, ${ }^{5}$ including an introduction to theoretical aspects of cyclic voltammetry as well as several practical learning modules. The authors present this guide for graduate-level selfinstruction, though it is also useful for teaching in upper-division courses.

Ultramicroelectrodes (UMEs) are electrodes for which at least one dimension is in the range between $0.1 \mu \mathrm{m}$ and $50 \mu \mathrm{m} .{ }^{8}$ Throughout this article, we will refer specifically to disk UMEs. This geometry presents a qualitative difference with respect to standard-sized electrodes: the geometry of the diffusion field presents an almost spherical symmetry. This translates in a great increase in the efficiency of the mass transport towards and from the electrode surface, i.e. an increase in the total amount of charge transferred per unit area. ${ }^{9}$ These systems are often introduced separately (or even overlooked) and the hemispherical diffusion regime is discussed..$^{7,8,10-13}$ This approach does not fully reflect the continuum that exists between possible electrode sizes. More importantly, it overlooks the problems of edge effects in macroscopic electrodes and the apparition of peaks due to linear diffusion in voltammograms on UMEs at very high sweep rates.

Edge effects are an increase in the experimental current compared to what would be expected from linear semi-infinite diffusion

\footnotetext{
*e-mail: fzanotto@fsu.edu
}

conditions, because concentration gradients with nonzero components in the $x-y$ plane are originated near the edges of the disk electrode. ${ }^{14-16}$ This current increase is proportional to the disk's perimeter instead of its area, for a chronoamperometric experiment. ${ }^{14}$

In cyclic voltammetry, edge effects become significant for long experiments (i.e. low sweep rates). Thus, it is very important to be aware of these issues in order to avoid them or take them into account when analyzing results. Consequently, the discussion of edge effects and UMEs is not only necessary, but also a good opportunity to discuss mass transport by diffusion, ${ }^{12,17-19}$ which often proves difficult to grasp, especially in 2D and 3D domains. Thus, the importance of the activity herein proposed is twofold: to highlight the importance of taking into account or minimizing edge effects in voltammetry, and to rationally and seamlessly explain the behavior of UMEs in terms of radial diffusion.

On another note, the use of computational modelling and simulation in electrochemistry is one more aspect often overlooked in electrochemistry courses and textbooks. Currently, modern computers allow the simulation of electrochemical experiments in 2D and 3D geometries in very short times. While the use of simulations is quite extended in electrochemical research, ${ }^{20-24}$ and some introductory texts can be used for reference, ${ }^{2,22,25}$ only a few articles about its usefulness in education in electrochemistry can be found..$^{26-31}$

We present here a hands-on approach that combines experiments and simulations to naturally introduce the concept of edge effects in macroscopic electrodes and hemispherical diffusion in UMEs. The main goal of this activity is to allow the discussion of some important aspects: diffusion in three-dimensional space, the differences between electrodes of different sizes and different sweep rates in voltammetry experiments, how these factors affect the voltammetric response and why. We propose to facilitate this process with the help of the visualization of concentration profiles from simulations that, in turn, have been previously validated by means of a direct comparison with experimental results.

At the end of this activity, students should be able to:

- Set up a simple electrochemical cell, taking into account safety 
concerns and electrode cleanliness.

- Understand how diffusion at the edges of electrodes differs from diffusion at the center of macroscopic electrodes.

- Understand how the electrochemical response of the electrodes can be explained in terms of this difference.

- Understand the diffusion fronts that are generated around an UME, and how they correlate to their voltammetric response.

- Acknowledge the value of computational simulations to obtain information often inaccessible in experiments.

- Acknowledge the importance of validating models by means of comparison with experimental results.

\section{EXPERIMENTS AND SIMULATIONS}

Less than three hours were necessary for the activity, including experiments, simulations and discussion. The activity involves interspersed experiments and simulations in order to allow for the results of each to feedback on the other. The Supplementary Material includes student handouts, the results for the data analysis detailing all the important concepts and conclusions to be drawn from the experiments, post-lab questions for students and a guide for instructors with details concerning the experiments and simulations.

\section{Experiments}

The experiment consisted of electrode preparation (mechanical polishing, electrochemical pretreatment), sparging with nitrogen gas and simple cyclic voltammetry measurements. A $5 \mathrm{mmol} \mathrm{L}^{-1}$ potassium ferricyanide in $0.5 \mathrm{~mol} \mathrm{~L}^{-1} \mathrm{KCl}$ solution was used, although any reversible, non-adsorbing electroactive species could work. The instructor can perform the pretreatment beforehand to save time. Three different gold electrodes, $10 \mu \mathrm{m}$ and $1 \mathrm{~mm}$ (Cypress Systems Inc. $)^{32}$ and $2 \mathrm{~mm}$ (CH Instruments model CHI101) in diameter were used in this activity. These sizes were chosen for their widespread availability, although other available sizes, such as $25 \mu \mathrm{m}, 1.6 \mathrm{~mm}$ and $3 \mathrm{~mm}$ can be used as replacements, respectively. ${ }^{33-35}$

An Autolab PGSTAT100 potentiostat-galvanostat was used to perform the experiments. Many accessible potentiostats are commercially available. ${ }^{36,37}$ Alternatively, low-cost tools for cyclic voltammetry can be assembled by following instructions found elsewhere..$^{38,39}$

\section{Hazards}

Standard lab safety measures should be in place for the experiments. Gloves, eye protection and adequate clothing should be used when preparing solutions and assembling the electrochemical cell. $\mathrm{H}_{2} \mathrm{SO}_{4}$ is highly corrosive and irritant when in contact with skin and eyes. Potassium ferricyanide is irritant in contact with skin and eyes. Potassium chloride is slightly irritant in contact with skin and eyes. Waste products containing $\mathrm{H}_{2} \mathrm{SO}_{4}$ and potassium ferricyanide should be treated and disposed of properly. Students should be instructed to not manipulate the electrochemical cell when a measurement is in progress in order to avoid electrical shock. The use of a Faraday cage is recommended.

\section{Simulations}

Finite element method simulations were carried out using the COMSOL Multiphysics software version 4.2a. This software package is ideal for the efficient modelling of electrochemical processes in two-dimensional systems, ${ }^{22}$ which is necessary to observe edge effects and hemispherical diffusion. A 2D axisymmetric model, representing a 3D system was set up before the activity (included in the Supplementary Material). This model considers mass transport only by diffusion and Butler-Volmer flux boundary conditions at the electrode surface. A large exchange current parameter was used to simulate a reversible electron transfer process. This project was optimized for ease of use and quick calculation. During the activity, only the sweep rate, electrode radius and time duration parameters need to be changed, and the necessary graphs are plotted by default and easily exported for further analysis and comparison (2D concentration maps, sweep rate-normalized voltammograms and area-normalized voltammograms). All running times were lower than two minutes in a standard desktop computer (intel i5 processor). The results were carefully checked for convergence..$^{40}$

Since this software can be cost prohibitive, the Supplementary Material contains all of the outputs necessary for the analysis and interpretation of the experimental results, including the most commonly available electrode sizes. Additionally, other methods for simulating cyclic voltammetry experiments, such as finite difference, can be used..$^{27,30}$ Examples of code for MATLAB can be found online ${ }^{41}$ and adapted for 2D systems.

\section{DISCUSSION}

The laboratory experiment herein proposed expands on traditional activities directed to introduce basic aspects of cyclic voltammetry to beginners, such as the ones proposed by Elgrishi et al. ${ }^{5}$ It is suggested that students acquire a basic theoretical background concerning voltammetry of simple one-electron reversible processes beforehand. Although the activity involves the use of UMEs, it is set up in order to gradually build an understanding of radial diffusion, posing questions and answering them by means of the interplay of experiments and simulations. Thus, previous specific knowledge about UMEs is not necessary. The insights from this activity can later be complemented by the discussion of other important aspects of this topic, such as the use of UMEs in low conductivity media. ${ }^{8}$

The activity is presented in its complete form in the Supplementary Material section. A brief summary is presented here. In a first step, simple voltammetry experiments are carried out. The students are asked to compare the voltammograms after dividing the current by the square root of the sweep rate (Figure 1.a). A difference in the shape of the voltammograms can be spotted, particularly noticeable after the negative current peak, and this encourages them to pose hypotheses that could explain this result. The simulations are then validated by comparison with the experimental result under identical conditions (Figure 1.b) and the simulated concentration maps are used to explain this outcome in terms of edge effects. The fact that the simulation is able to predict this difference indicates that it should be explained it in terms of the simple physical process that the model is based upon (i.e. diffusion or reversible charge transfer). Later, the students perform an experiment using an UME, and are asked to explain the shape of the voltammogram in terms of the previous results. Finally, a simulation is carried out under conditions that are not experimentally attainable, in order to demonstrate both the predictive power of the concepts that were acquired during the activity and the usefulness of simulations.

During this activity, the current response corresponding to three different diffusion regimes are observed both experimentally and in simulations, illustrating the continuum that exists between macroscopic electrodes and UMEs, and its dependence on sweep rate for voltammograms. A simple test can be performed a priori to evaluate whether edge effects will be observable for a given experiment, by comparing the electrode diameter with the square root of the mean square displacement in $1 \mathrm{D}$, which is a measure of the length of the diffusion layer: 

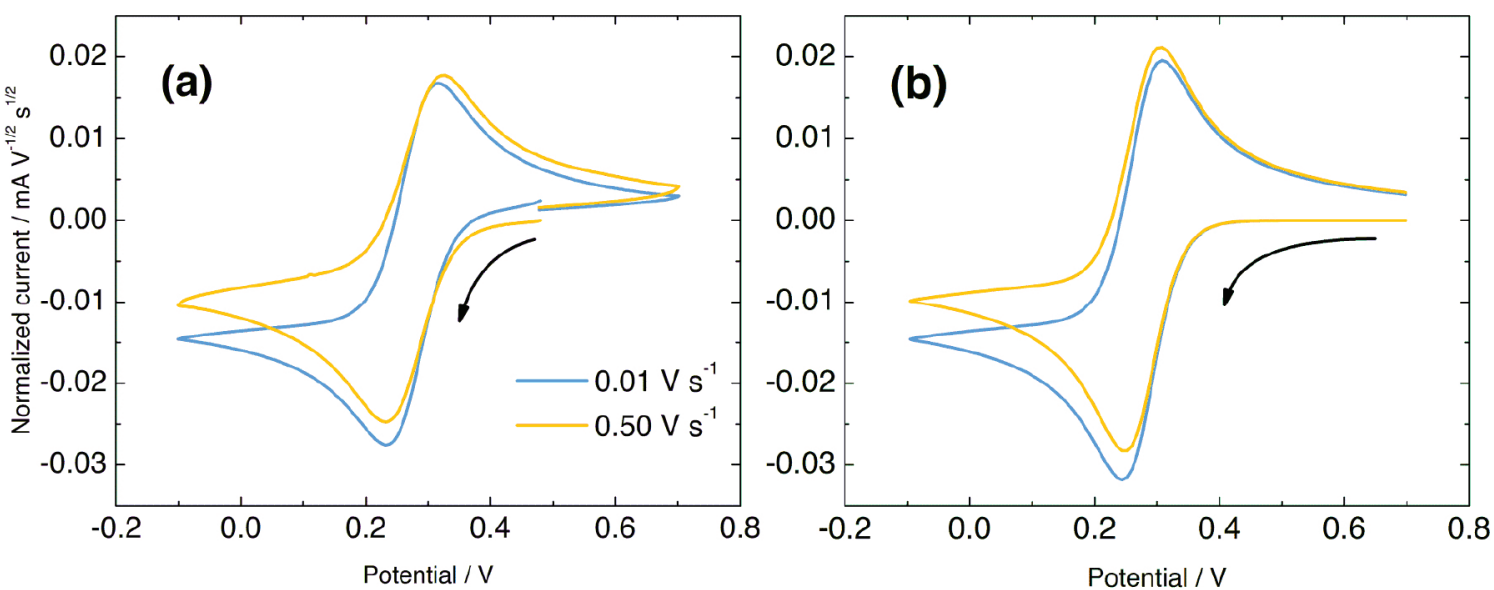

Figure 1. Experimental (a) and simulated (b) normalized cyclic voltammograms for a gold electrode $1.0 \mathrm{~mm}$ in diameter at two different sweep rates. A deviation is observed for a sweep rate of $0.01 \mathrm{~V} \mathrm{~s}^{-1}$, due to the increased weight of edge effects. Simulation parameters: Diffusion coefficient for reagent: $6.67 \times 10^{-10} \mathrm{~m}^{2} \mathrm{~s}^{-1}$, for product: : $7.18 \times 10^{-10} \mathrm{~m}^{2} \mathrm{~s}^{-1}$, standard charge transfer potential: $0.275 \mathrm{~V}$, vertex potential: $-0.100 \mathrm{~V}$, initial potential: $0.700 \mathrm{~V}$, initial concentration of reagent: $5.00 \mathrm{mmol} \mathrm{L}^{-1}$

$$
\sqrt{\langle x\rangle}=\sqrt{2 D t}
$$

where $\mathrm{D}$ is the diffusion coefficient of the reactant species and $\mathrm{t}$ is the dime duration of the experiment, which can be calculated as:

$$
t=2 \frac{\text { Vertex potential }- \text { Initial potential }}{\text { scan rate }}
$$

For a typical scan rate of $0.100 \mathrm{~V} \mathrm{~s}^{-1}$ and a diffusion coefficient of $6.67 \times 10^{-10} \mathrm{~m}^{2} \mathrm{~s}^{-1}$, Equation (1) yields $\sqrt{\langle x\rangle}=0.137 \mathrm{~mm}$. This value is significantly lower than $1.00 \mathrm{~mm}$ and $2.00 \mathrm{~mm}$, thus no edge effects are expected to be noticeable for these electrodes at $0.100 \mathrm{~V} \mathrm{~s}^{-1}$. The UME, however, is expected to show a very different current response, since $25 \mu \mathrm{m}<<0.137 \mathrm{~mm}$. For the lowest scan rates used in the activity, $0.010 \mathrm{~V} \mathrm{~s}^{-1}, \sqrt{\langle x\rangle}=0.432 \mathrm{~mm}$, which is much closer to the electrode diameter values of $1 \mathrm{~mm}$ and $2 \mathrm{~mm}$, and thus edge effects are to be expected. This analysis can be performed with students before performing experiments during the activity, though the visual nature the simulation results is clearer.

Concentration maps corresponding to the vertex potential of the scan (Figure 2) are a very helpful way of visualizing and interpreting these results and thus are used extensively as a tool to interpret the experimental and simulated current responses. Figure $2 \mathrm{a}$ shows a typical profile for which the linear diffusion regime is prevalent, corresponding to large surface area and high scan rate (short experiment duration). In this case, the typical reversible diffusional voltammogram is experimentally observed. Figure $2 b$ shows the same results for $a$ slightly smaller electrode radius and a larger experiment duration. In this situation, the edges of the diffusion front are noticeably curved, this effect can be noticed in the current response. Finally, Figure 2c shows the concentrations in the vicinity of a UME, for which the isoconcentration lines are represented by semicircles. After this activity, students should be able to rationalize the experimental signal for a UME in cyclic voltammetry from this figure.

This activity has been performed as part of our electrochemistry undergraduate course, in which students worked in groups of approximately 8 , due to limited availability of the potentiostat, and also as training for undergraduate students joining our group.

Students reported that the simulation results greatly helped their visualization of the physical processes taking place in the cell. The possibility of performing simulations during the activity extended their comprehension of the static plots that are found in books and

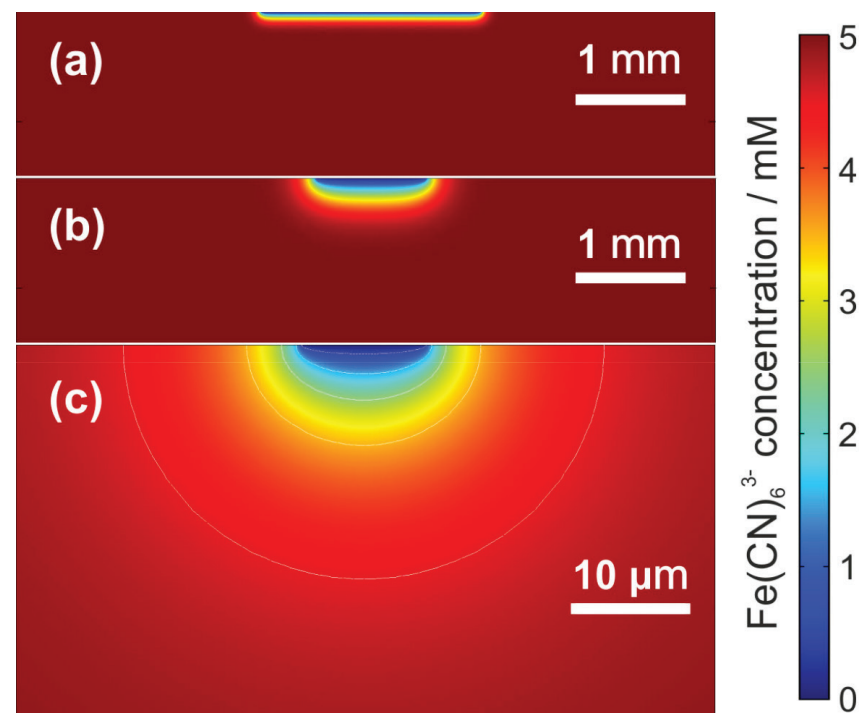

Figure 2. Concentration maps for the oxidized species extracted from simulations at the switching potential $(-0.1 \mathrm{~V})$ for different electrode diameter $(d)$ and sweep rate (v). (a) $d=2.0 \mathrm{~mm}, v=0.10 \mathrm{~V} \mathrm{~s}^{-1}$. (b) $d=1.0 \mathrm{~mm}, v=0.01 \mathrm{~V} \mathrm{~s}^{-1}$. (c) $d=10.0 \mu \mathrm{m}, v=0.50 \mathrm{~V} \mathrm{~s}^{-1}$. Instructions for obtaining these plots are detailed in the Supplementary Material

allowed them to correlate the current response with the different shapes of the concentration profiles that develop near the electrodes. They were able to infer for themselves which are the important factors affecting the experimental results and later correctly infer by extrapolation the shape of the voltammogram for the UME. Analysis of the answers to the questions posed by the instructors during the activity and of the post-lab questions shows that most of them understood the interplay between linear and hemispherical diffusion regimes, their influence in the behavior of UMEs and large planar electrodes and the continuum that exists between them, as a function of experiment duration, without the need for a rigorous theoretical introduction.

\section{CONCLUSIONS}

The relationship between edge effects and hemispherical diffusion is an often-overlooked aspect in education in electrochemistry. The 
approach herein proposed (detailed in the Supplementary Material) attends to this issue. The possibility of performing both simulations and experiments in which results are directly contrastable is an effective pedagogical tool. While experiments provide the students with interesting questions and challenge their expectations, simulations offer additional information that explain the results. In turn, new experiments are conceived that confirm the explanation. This approach has been effective in seamlessly introducing new concepts and is highly transferable for the study of many other aspects in electrochemistry.

\section{SUPPLEMENTARY MATERIAL}

The Supplementary Material is available on http://quimicanova. sbq.org.br, with free access. The following files are included:

- Student handout, results and analysis of questions in handout, notes for instructors and post-lab questions (DOCX).

- Simulations results, containing voltammogram TXT files and concentration maps PNG files (ZIP).

- COMSOL Multiphysics projects (MPH).

\section{ACKNOWLEDGEMENTS}

FMZ thanks CONICET for the fellowship granted. Financial support from CONICET, Secretaría de Ciencia y Tecnología de la Universidad Nacional de Córdoba (SECyT-UNC) and Fondo para la Investigación Científica y Tecnológica (FONCyT) PICT-2012-1820 are gratefully acknowledged. The authors thank F. Cometto, S. Dassie, A. Fernández and M. Taccone and for their helpful advice.

\section{REFERENCES}

1. Kissinger, P. T.; Heineman, W. R.; J. Chem. Educ. 1983, 60, 702.

2. Compton, R. G.; Banks, C. E.; Understanding Voltammetry, $2^{\text {nd }}$ ed., Imperial College Press, Singapore, London, 2010.

3. Scholz, F.; Electroanalytical Methods: Guide to Experiments and Applications, $2^{\text {nd }}$ ed., Springer-Verlag: Berlin, Heidelberg, 2010.

4. Van Benschoten, J. J.; Lewis, J. Y.; Heineman, W. R.; Roston, D. A.; Kissinger, P. T.; J. Chem. Educ. 1983, 60, 772.

5. Elgrishi, N.; Rountree, K. J.; McCarthy, B. D.; Rountree, E. S.; Eisenhart, T. T.; Dempsey, J. L.; J. Chem. Educ. 2018, 95, 197.

6. Mabbott, G.; J. Chem. Educ. 1983, 60, 697.

7. Bard, A. J.; Faulkner, C. R.; Electrochemical Methods, Fundamentals and Applications, $2^{\text {nd }}$ ed., John Wiley \& Sons: New York, 2001.

8. Montenegro, M. I.; Arlete Queirós, M.; Daschbach, J. L.; Microelectrodes: Theory and Applications, Kluwer Academic: Dordrecht, Boston, 1991.

9. Heinze, J.; Berichte der Bunsengesellschaft für Phys. Chemie 1981, 85, 1096.
10. Gileadi, E.; Electrode Kinetics for Chemists, Chemical Engineers and Materials Scientists, $1^{\text {st }}$ ed., John Wiley \& Sons, New York, 1993.

11. Eliaz, N.; Gileadi, E.; Physical Electrochemistry Fundamentals, Techniques and Applications, Wiley-VCH: Weinheim, 2011.

12. Ching, S.; Dudek, R.; Tabet, E.; J. Chem. Educ. 1994, 71, 602.

13. Holze, R.; Experimental Electrochemistry: A Laboratory Textbook, Second; Wiley-VCH: Weinheim, 2017.

14. Oldham, K. B.; J. Electroanal. Chem. 1981, 122, 1

15. Speiser, B.; Pons, S.; Can. J. Chem. 1982, 60, 1352.

16. Speiser, B.; Pons, S.; We, I.; Can. J. Chem. 1982, 60, 2463.

17. Anderson, L. B.; Reilly, C. N.; J. Chem. Educ. 1967, 44, 9.

18. Palombari, R.; J. Chem. Educ. 1992, 69, 473.

19. Gomez, M. E.; Kaifer, A. E.; J. Chem. Educ. 1992, 69, 502.

20. Gavaghan, D. J.; Gillow, K.; Süli, E.; Langmuir 2006, 22, 10666.

21. Gooch, K. A.; Qiu, F. L.; Fisher, A. C.; The Digital Simulation of Voltammetry under Stagnant and Hydrodynamic Conditions. In Encyclopedia of Electrochemistry; Wiley-VCH Verlag $\mathrm{GmbH} \& \mathrm{Co}$. KGaA: Weinheim, 2007.

22. Roger W. Pryor.; Multiphysics Modeling Using COMSOL: A First Principles Approach, $1^{\text {st }}$ ed., Jones and Bartlett Publishers, USA, 2011.

23. Dickinson, E. J. F.; Ekström, H.; Fontes, E.; Electrochem. Commun. 2014, 40, 71.

24. Peljo, P.; Scanlon, M. D.; Stockmann, T. J.; Curr. Opin. Electrochem. 2018, 7, 200.

25. Britz, D.; Digital Simulation in Electrochemistry; Lecture Notes in Physics, Springer Berlin Heidelberg: Berlin, Heidelberg, 2005.

26. Sanchez, G.; Codina, G.; Aldaz, A.; J. Chem. Educ. 1991, 68, 489.

27. Prospisil, L.; Zalis, S.; Fanelli, N.; J. Chem. Educ. 1995, 72, 997.

28. Howard, E.; Cassidy, J.; J. Chem. Educ. 2000, 77, 409.

29. Messersmith, S. J.; J. Chem. Educ. 2014, 91, 1498.

30. Brown, J. H.; J. Chem. Educ. 2015, 92, 1490.

31. Bozzini, B. P.; J. Chem. Educ. 2000, 77, 100

32. Cypress Systems Inc. is no longer on the market.

33. https://www.edaq.com/Disk Electrodes, accessed June 2020

34. https://www.chinstruments.com/accessories.shtml, accessed June 2020.

35. https://www.basinc.com/products/ec/sve, accessed June 2020.

36. http://www.dropsens.com/en/potentiostats_pag.html\#stat8000p, accessed June 2020.

37. https://www.edaq.com/ER461, accessed June 2020.

38. Mott, J. R.; Munson, P. J.; Kreuter, R. A.; Chohan, B. S.; Sykes, D. G.; J. Chem. Educ. 2014, 91, 1028.

39. Li, Y. C.; Melenbrink, E. L.; Cordonier, G. J.; Boggs, C.; Khan, A.; Isaac, M. K.; Nkhonjera, L. K.; Bahati, D.; Billinge, S. J.; Haile, S. M.; Kreuter, R. A.; Crable, R. M.; Mallouk, T. E.; J. Chem. Educ. 2018, 95, 1658.

40. Cutress, I. J.; Dickinson, E. J. F.; Compton, R. G.; J. Electroanal. Chem. 2010, 638, 76 .

41. https://la.mathworks.com/matlabcentral/fileexchange/64011-cyclicvoltammetry-simulator, accessed June 2020. 